\title{
Longitudinal Magnetostriction and Its Dependence on the Method of Demagnetization in Single Crystals and a Polycrystal of 0.5 Percent Aluminium Iron, IV. The [210] Single Crystal and a Polycrystal ${ }^{*}$
}

\author{
By Ryôfu Miyasawa**
}

\begin{abstract}
The longitudinal magnetostriction has been studied at ordinary temperatures in the thermally demagnetized state and in the alternating-current demagnetized state with two rod specimens of iron containing $0.5 \% \mathrm{Al}$, using the optical lever method. One of the specimens is a single crystal whose rod axis is situated between [100] and [110], called the [210] specimen, and the other a polycrystal made of the raw material used for the preparation of single crystals. It has been found that the magnetostrictive behavior of the [210] specimen is as a whole intermediate between the behaviors of the [100] and [110] specimens, and consequently shows a strong dependence on the method of demagnetization. On the contrary, it has been noted that the magnetostrictive behavior of the polycrystal specimen is as a whole similar to that of the [110] specimen in a thermally demagnetized state, but there is a considerable difference between them in that the dependence of the magnetostrictive behavior on the method of demagnetization in the former is far less than in the latter. Furthermore, in both the [210] specimen and the polycrystal the difference of magnetostriction between the state of remanent magnetization and that of magnetic saturation is independent of the method of demagnetization. In addition, the value of the magnetostrictive constant, $\lambda_{111}$, of these specimens is $-20.5 \times 10^{-6}$, as determined in consideration of the difference of magnetostriction between the two states described above.
\end{abstract}

(Received January 22, 1969)

\section{Introduction}

Following the preceding papers ${ }^{(1)} \sim(3)$ on the [100], [110] and [111] rod single crystals of iron containing $0.53 \% \mathrm{Al}$, the present study deals with the results of measurements on the longitudinal magnetostriction and their analyses of the [210] rod single crystal and a polycrystal carried out in the same way as mentioned in the preceding study(1). The single crystal specimen produced by the same method and from the same material as used

Table 1 Orientation of the [210] single crystal rod specimen, and dimensions of the single crystal and polycrystal rod specimens, $0.53 \%$ aluminium iron

\begin{tabular}{|c|c|c|c|c|c|c|}
\hline \multicolumn{2}{|c|}{ Specimen } & \multirow{2}{*}{\multicolumn{3}{|c|}{$\begin{array}{l}\text { Direction cosines of the rod } \\
\text { axis referred to the crystal } \\
\text { axis, or grain size }\end{array}$}} & \multirow{3}{*}{$\begin{array}{c}\begin{array}{c}\text { Length } \\
(\mathrm{cm})\end{array} \\
6.096\end{array}$} & \multirow{3}{*}{$\frac{\begin{array}{c}\text { Diameter } \\
(\mathrm{cm})\end{array}}{0.2566}$} \\
\hline Group & No. & & & & & \\
\hline [210] & 10 & $\beta_{1}=0.919$ & $\beta_{2}=0.392$ & $\beta_{3}=0.040$ & & \\
\hline Polycrystal & 11 & \multicolumn{3}{|c|}{ Grain size $140 \mathrm{~mm}^{-2}$} & 6.904 & 0.2530 \\
\hline
\end{tabular}

in the preceding paper ${ }^{(1)}$ has a crystallographic orientation intermediate between $[100]$ and $[110]$, as seen in Table 1 , where the direction cosines of the rod axis referred to the cubic axes, denoted by $\beta_{1}, \beta_{2}$ and $\beta_{3}$, are given, with its dimensions. The polycrystal specimen is a cylindrical bar made of the same material as used for the preparation of single crystals and subjected to the decarburization treatment, which is a prerequisite procedure for the strainanneal method to realize grain growth. Its grain size and dimensions are also given in Table 1. Furthermore, important data on the magnetization of these specimens are given in Table 2.

\section{Magnetostriction of the [210] Specimen}

The main data on the longitudinal magnetostriction $\lambda^{t}$ of the [210] specimen, No. 10, after TD and AD are given in Table 3 , and the $\lambda^{t}-I^{m}$ curve and the $\lambda^{t}-H^{m}$ curve after TD are shown in Figs. 1 and 2, respectively, together

Table 2 Measured data on the saturation magnetization $\left(I_{s}\right)$, magnetization corresponding to the break of the descending hysteresis curve $\left(I_{k}^{m}\right)$, coercive force $\left(H_{c}\right)$ and initial susceptibility $\left(X_{0}\right)$ in two different demagnetized states

\begin{tabular}{c|c|c|c|c|c|c|c|c|}
\hline \hline \multirow{2}{*}{$\begin{array}{c}\text { Specimen } \\
\text { No. }\end{array}$} & \multicolumn{4}{|c|}{ Thermally demagnetized (TD) state } & \multicolumn{4}{c|}{ Alternating-current demagnetized (AD) state } \\
\cline { 2 - 9 } & $I_{s}$ & $I_{k}^{m}$ & $H_{c}(\mathrm{Oe})$ & $\chi_{0}$ & $I_{s}$ & $I_{k}^{m}$ & $H_{c}(\mathrm{Oe})$ & $\chi_{0}$ \\
\hline 10 & 1700 & 1237 & -0.55 & 22.0 & 1701 & 1237 & -0.52 & 16.1 \\
11 & 1678 & 1164 & -1.04 & 59.2 & 1688 & 1197 & -0.99 & 32.8 \\
\hline
\end{tabular}

* The original of this paper, written in Japanese was published previously in J. Japan Inst. Metals, 31 (1967), 1223.

** The Faculty of Science, Hirosaki University, Hirosaki, Japan
(1) R. Miyasawa : Trans. JM, 10 (1969), 320.

(2) R. Miyasawa : Trans. JIM, $10(1969), 328$.

(3) R. Miyasawa : Trans. JIM, 10 (1969), 335. 
with the $I^{m}-H^{m}$ curves. As anticipated, the magnetostrictive behavior of the [210] specimen is intermediate between the behaviors of the $[100]$ and $[110]$ speci-

Table 3 Measured data on the longitudinal magnetostriction of the [210] single crystal specimen, No. 10; the values corresponding to the states of saturation, remanent magnetization and coercive force are denoted by $\lambda_{s}, \lambda_{k}^{t}$ and $\lambda_{c}^{t}$, respectively

\begin{tabular}{c|c|c|c|c}
\hline $\begin{array}{c}\text { Demagnetized } \\
\text { state }\end{array}$ & $\begin{array}{c}\lambda_{s} \\
\times 10^{6}\end{array}$ & $\begin{array}{c}\lambda_{k}^{t} \\
\times 10^{6}\end{array}$ & $\begin{array}{c}\lambda_{c}^{t} \\
\times 10^{6}\end{array}$ & $\begin{array}{c}\left(\lambda_{k}^{t}-\lambda_{s}\right) \\
\times 10^{6}\end{array}$ \\
\hline TD & 5.1 & 9.0 & 12.7 & 3.9 \\
AD & -9.1 & -5.1 & -1.5 & 4.0 \\
\hline
\end{tabular}

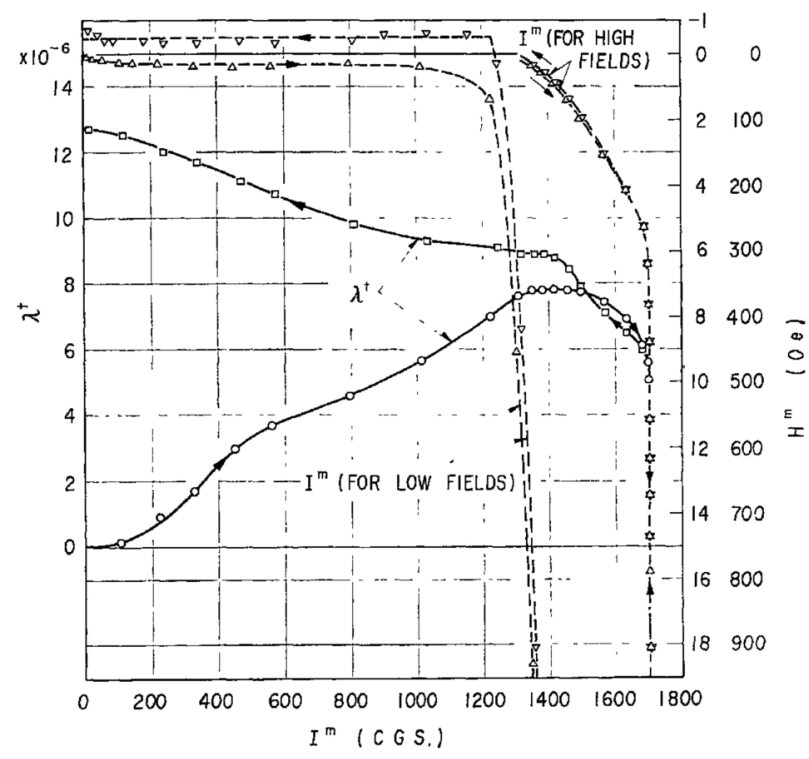

Fig. $1 \lambda^{t}-I^{m}$ curve in the thermally demagnetized state of the [210] specimen, No. 10. $I^{m}-H^{m}$ curves for low and high fields are also shown

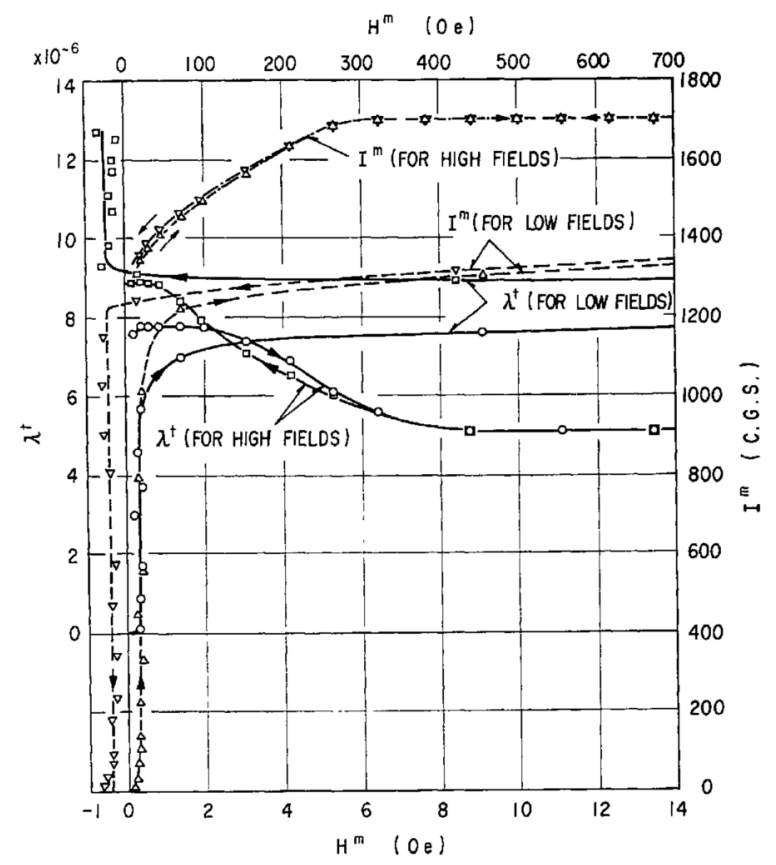

Fig. $2 \lambda^{t}-H^{m}$ curves for low and high fields in the thermally demagnetized state of the [210] specimen, No.10. Corresponding $I^{m}-H^{m}$ curves are also shown mens. Namely, the virgin curve of magnetostriction of this specimen in the range of wall displacements closely resembles that of the $[100]$ specimen $^{(1)}$, except that the magnitude of magnetostriction of the former is smaller than that of the latter. Therefore, it is considered that in this range $90^{\circ}$ wall displacements distinctly contribute to the magnetostriction, as in the case of the [100] specimen. With further increase in magnetization, a flat maximum takes place in the $\lambda^{t}-l^{m}$ curve near the state corresponding to remanencebecause of contraction in the middle portion of the specimen due to the rotation process, as in the case of the [110] specimen, and subsequently a continuous contraction occurs until saturation. Furthermore, it is likely that the elongation due to irreversible $90^{\circ}$ wall displacements in the ends of the specimen occurs simultaneously in the range of rotation, as is the case with the [100] specimen. This may be understood from the experimental fact that the descending hysteresis curve of magnetostriction is immediately detached from the virgin curve, when the magnetization is decreased from saturation. That is, although the rotation process in the middle portion of the specimen retraces the initial steps, $90^{\circ}$ wall displacements in the end portions are irreversible, leading to lag of the decrease in magnetization. When the magnetization is decreased from saturation, the magnetostriction recovers in the aforementioned way, and the descending hysteresis curve of magnetostriction intersects upwards the virgin curve in the midst of the range of rotation, and subsequently, after suspension of change near the remanence point, the specimen again continues to elongate up to the coercive force point. Since the descending hysteresis curve of magnetostriction in the range of wall displacements described above resembles that of the [100] specimen except for the difference of magnitude of the magnetostriction, it appears that the magnetostriction in this range originates in $90^{\circ}$ wall displacements as well as in $180^{\circ}$ wall displacements, and that the domain distribution at the coercive force point can also be expressed for the [210] specimen by eq. (16) of the preceding paper ${ }^{(1)}$.

Next, the $\lambda^{t}-I^{m}$ and $\lambda^{t}-H^{m}$ curves of the [210] specimen after $\mathrm{AD}$ are shown in Figs. 3 and 4, respectively, with the $I^{m}-H^{m}$ curves. As seen in the $\lambda^{t}-I^{m}$ curve, the magnetostriction after $\mathrm{AD}$, which has no positive value in the whole range of magnetization, is markedly different from that after TD, as in the case of the[ 100$]$ and [110] specimens ; namely, the negative magnetostriction arises at an extremely low value of magnetization (about 50 C.G.S.), and its absolute value continues to increase along a nearly linear path until the state corresponding to remanence is attained, and subsequently, after suspension of its increase near the state described just above, it again continues to increase monotonically to saturation. When the magnetization is decreased from saturation, the descending hysteresis curve of magnetostriction almost completely retraces the virgin curve in the range of rotation, as seen in Fig. 3, but in the range of wall displacements the magnetostriction curve does not recover to the virgin curve, although the forms of the $\lambda^{t}-I^{m}$ curves resemble each other. In addition, a comparison between Tables 3 and 4 shows that the measured values of $\lambda_{k}^{t}-\lambda_{s}$ 
and $\lambda_{c}^{t}-\lambda_{s}$ of the [210] specimen after $\mathrm{AD}$ are almost equivalent to those after TD, respectively.

From the above considerations it seems that the domain distribution in the unmagnetized state after AD can be expressed by eq. (16) of the preceding paper ${ }^{(1)}$, as in the case of the [100], [110] and [111] specimens. It is also considered that from the relations of the direction cosines for the [210] specimen, i.e. $\beta_{1}>\beta_{2}>\beta_{3} \simeq 0$, the following equations hold :

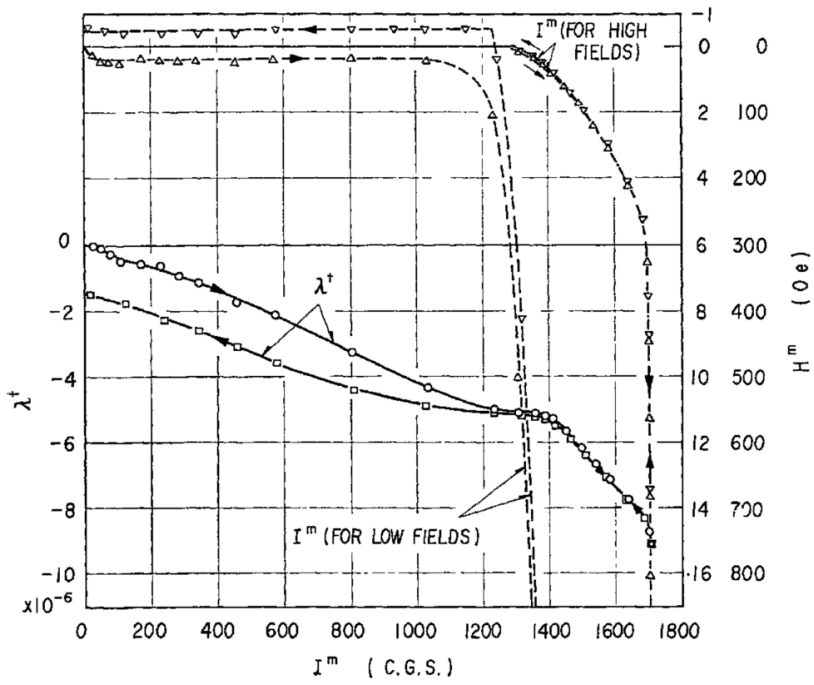

Fig. $3 \lambda^{t}-I^{m}$ curve in the alternating-current demagnetized state of the [210] specimen, No. 10. $I^{m}-H^{m}$ curves for low and high fields are also shown

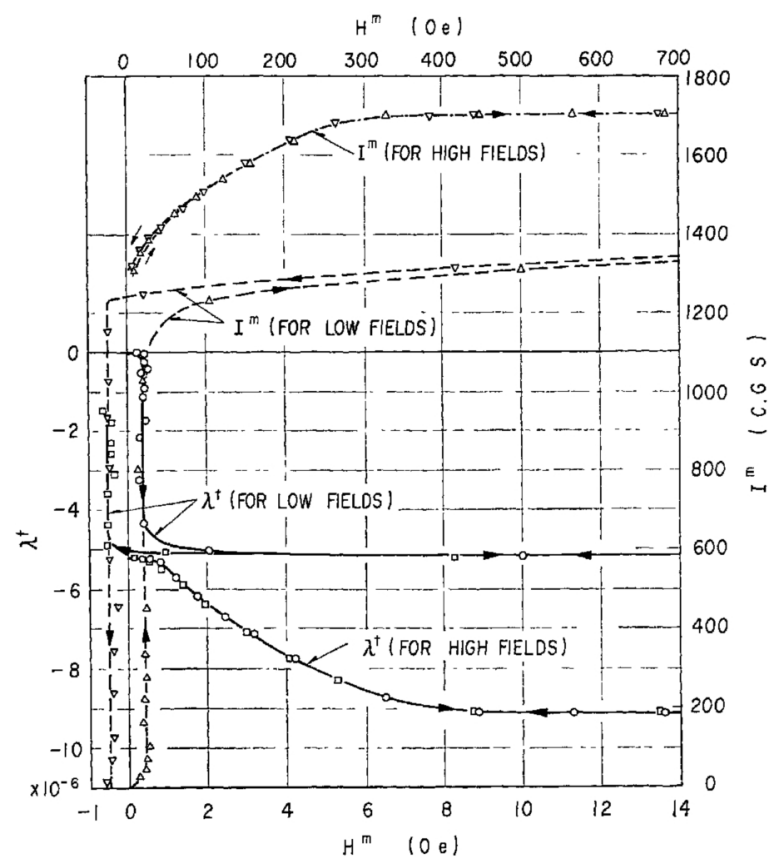

Fig. $4 \lambda^{t}-H^{m}$ curves for low and high fields in the alternating current demagnetized state of the [210] specimen, No. 10. Corresponding $I^{m}-H^{m}$ curves are also shown

$$
V_{1 c}=V_{\overline{1} c}>V_{2 c}=V_{\overline{2} c} \text { and } V_{3 c}=V_{\overline{3} c} \simeq 0 \text {. }
$$

In these equations $V_{i c}$ 's denote the relative volumes of the domains in the unmagnetized state whose directions of the magnetization vectors are shown by $i$ 's, where $i$ 's $=1, \bar{I}$,

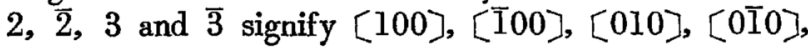

$[001]$ and [00I], respectively. Now, supposing that, after Akulov's assumption (4) for pure iron, the magnetization of the [210] specimen in the range of wall displacements proceeds at first by $180^{\circ}$ wall displacements and then, after completion of them, by $90^{\circ}$ wall displacements, we cannot explain that the negative magnetostriction arises at such a low value of magnetization as seen in Fig. 3. For this end it is required to consider the contribution of $90^{\circ}$ wall displacements. Namely, it can be conceived that over the whole range of wall displacements, $90^{\circ}$ wall displacements symbolized by $V_{\overline{1}} \rightarrow V_{2}$ and $V_{\overline{2}} \rightarrow V_{1}$ contribute to the magnetization; the former is accompanied with a contraction of the specimen, and the latter with an elongation. Since, regarding the relative volumes of the [210] specimen in the unmagnetized state, the relation symbolized by $V_{\overline{1} c}>V_{\overline{\mathrm{z}} c}$ holds from eq. (1), it is expected that the contribution of $V_{\overline{1}} \rightarrow V_{2}$ to the magnetization is preferable to that of $V_{\overline{2}} \rightarrow V_{1}$, and so the negative magnetostriction arises in that magnetization range.

Moreover, such $90^{\circ}$ wall displacements as described above probably occur in the single crystal specimens after AD which have the three principal crystallographic orientations as well as different kinds of wall displacements. However, in the [110] and [111] specimens their contribution to the magnetostriction is thought to be very little on account of the symmetry of the cubic axes with respect to the rod axis. It may also be understood for the same reason that for the $[100]$ specimen after $\mathrm{AD}$ the negative magnetostriction in the range of wall displacements arises more distinctly than for the [110] and [111] specimens after $\mathrm{AD}$.

\section{Magnetostriction of Polycrystal Specimens}

As for the magnetostrictive behaviors of the polycrystal specimens, the main data on the longitudinal magnetostriction of specimen No. 11, as an example, after TD and AD are given in Table 4 , and the $\lambda^{t}-I^{m}$ curve and the $\lambda^{t}-H^{m}$

Table 4 Measured data on the longitudinal magnetostriction of the polycrystal specimen, No. 11; the same notations as adopted in Table 3 are used

\begin{tabular}{c|c|c|c|c}
$\begin{array}{c}\text { Demagnetized } \\
\text { state }\end{array}$ & $\begin{array}{c}\lambda_{s} \\
\times 10^{6}\end{array}$ & $\begin{array}{c}\lambda_{k}^{t} \\
\times 10^{6}\end{array}$ & $\begin{array}{c}\lambda_{c}^{t} \\
\times 10^{6}\end{array}$ & $\begin{array}{c}\left(\lambda_{k}^{t}-\lambda_{s}\right) \\
\times 10^{6}\end{array}$ \\
\hline $\mathrm{TD}$ & -7.5 & 2.7 & 0.8 & 10.2 \\
$\mathrm{AD}$ & -8.7 & 1.9 & 0.1 & 10.6 \\
\hline
\end{tabular}

curve after TD are shown in Figs. 5 and 6, respectively, together with the $I^{m}-H^{m}$ curves. It is obvious from Fig. 5 that the virgin curve of magnetostriction of the polycrystal specimen after TD resembles as a whole that of the $[110]$ specimen $^{(2)}$ after TD, except that the maximum value of magnetostriction of the former near the state corresponding to remanence is smaller than that of the latter. When the magnetization is decreased from saturation, the descending hysteresis curve of magnetostriction almost completely retraces the virgin curve in the range

(4) N. Akulov : Z. Phys., 69 (1931), 78. 


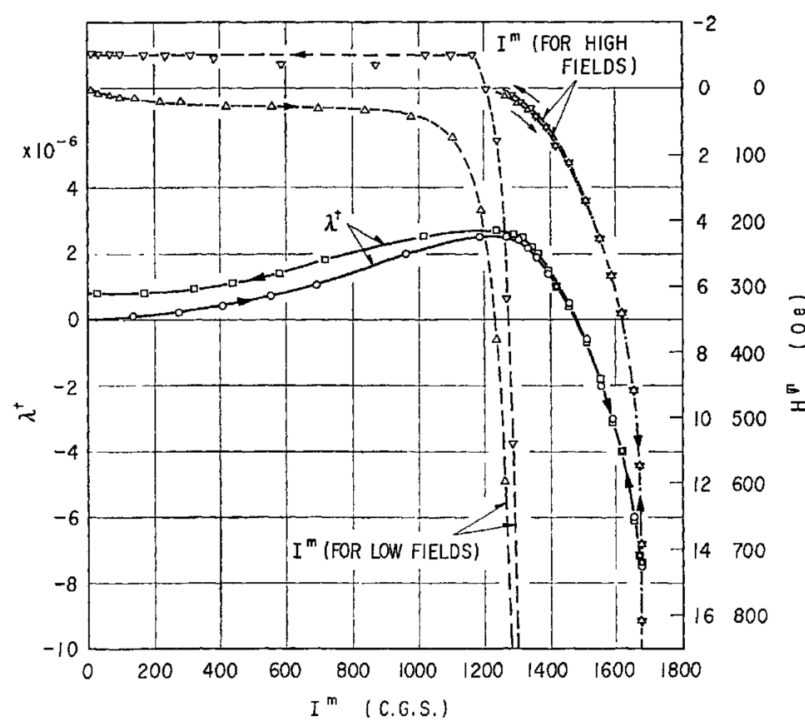

Fig. $5 \lambda^{t}-I^{m}$ curve in the thermally demagnetized state of the polycrystal specimen, No. 11. $I^{m}-H^{m}$ curves for low and high fields are also shown

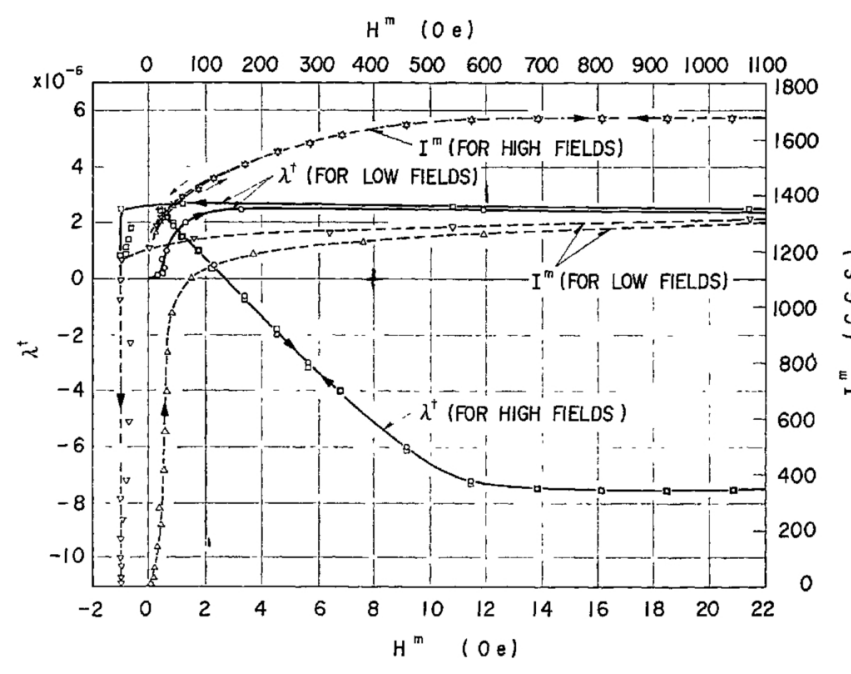

Fig. $6 \lambda^{t}-H^{m}$ curves for low and high fields in the thermally demagnetized state of the polycrystal specimen, No. 11. Corresponding $I^{m}-H^{m}$ curves are also shown

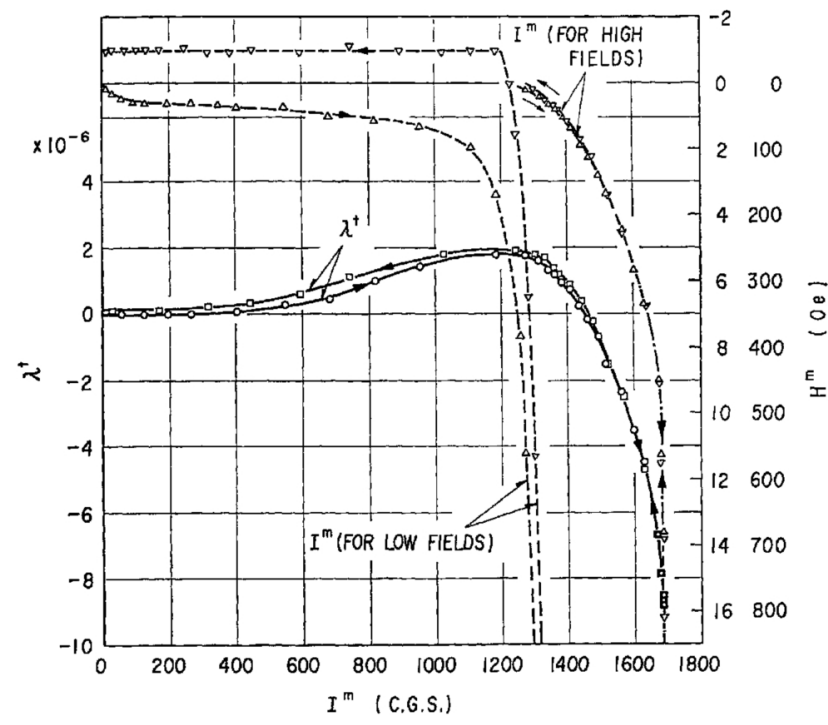

Fig. $7 \lambda^{t}-I^{m}$ curve in the alternating-current demagnetized state of the polycrystal specimen, No. $11 . \quad I^{m}-H^{m}$ curves for low and high fields are also shown

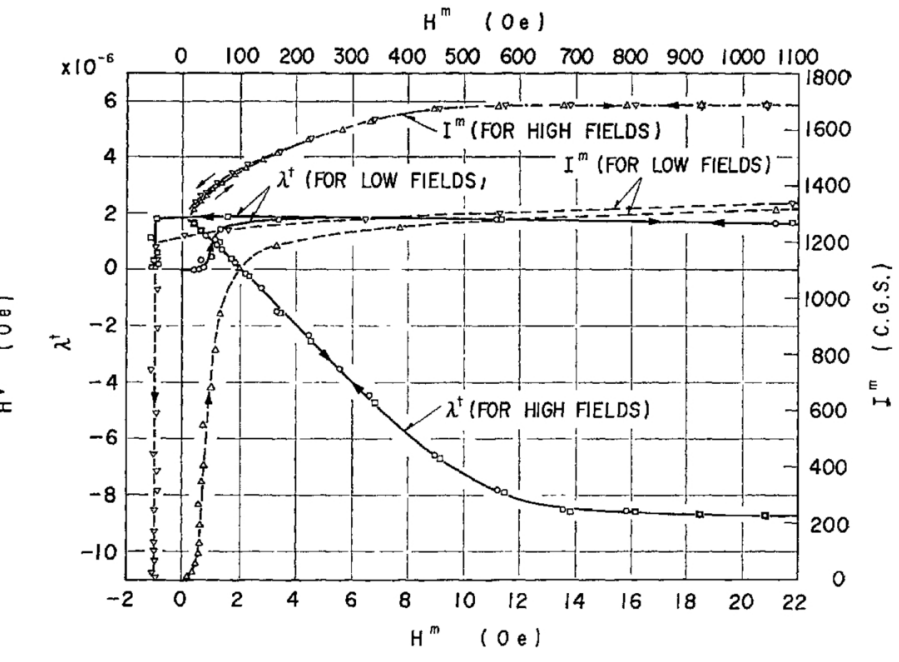

Fig. $8 \lambda^{t}-H^{m}$ curves for low and high fields in the alternatingcurrent demagnetized state of the polycrystal specimen, No.11. Corresponding $I^{m}-H^{m}$ curves are also shown

of rotation, but deviates upwards, showing a form analogous to the virgin curve, from the state near the remanence point to the coercive force point. Thus, there is a marked difference in the magnetostrictive behavior in the above range of wall displacements between the polycrystal specimen and the [110]specimen; namely, the latter has no contraction in that range in contrast with the former.

Next, the $\lambda^{t}-I^{m}$ curve and the $\lambda^{t}-H^{m}$ curve of the polycrystal specimen, No. 11, after AD are shown in Figs. 7 and 8 , respectively, together with the $I^{m}-H^{m}$ curves.

E A comparison of the $\lambda^{t}-I^{m}$ curves after TD and AD shown in Figs. 5 and 7 , indicates that the dependence of the magnetostrictive behavior on the method of demagnetization in the polycrystal specimen is far less than that in the single crystal specimens ; namely, the magnetostrictive behavior of the polycrystal specimen after $\mathrm{AD}$ closely resembles that after $\mathrm{TD}$, except that the absolute value of saturation magnetostriction in the former is larger than that in the latter and there is a difference between the maximum values of the positive magnetostriction near the remanence points (i.e. AD : $1.8 \times 10^{-6}$, TD : $2.5 \times 10^{-6}$ ). Furthermore, it is noted that the descending hysteresis curve of magnetostriction of the polycrystal specimen after $A D$ retraces the virgin curve more closely than that after $\mathrm{TD}$, so that the magnetostriction after AD scarcely remain at the coercive force point. Thus, the uniformity of the domain distribution in the AD state is inferior to that in the TD state, that is, the relative volumes in the $\mathrm{AD}$ state whose directions of magnetization vectors are close to the rod axis of the specimen are larger than those in the TD state, as in the case of the single crystal specimens. Moreover, as seen in Figs. 6 and 8, irrespective of the method of demagnetization, the descending hysteresis part of the $\lambda^{i}-H^{n}$ curve of the polycrystal specimen exactly coincides with the virgin curve in the range of rotation, and is almost linear in the range from a fairly weak field to the field just before saturation, as in the case of the [110] and $[111]$ specimens.

In addition, Tables 3 and 4 indicate that the difference of magnetostriction after $\mathrm{AD}$ between the remanence point and the saturation state, $\lambda_{k}^{t}-\lambda_{s}$, and that between the 
coercive force point and the saturation state, $\lambda_{c}^{t}-\lambda_{s}$, are almost equivalent to the respective values after TD. The above-mentioned experimental fact that the values of $\lambda_{k}^{t}-\lambda_{s}$ and $\lambda_{c}^{t}-\lambda_{s}$ are independent of the method of demagnetization has already been verified for the [100], [110], [111] and [210] specimens; therefore, it may safely be said that such a description holds for the single crystals and polycrystals investigated.

\section{Determination of the Magneto- strictive Constants}

The value of the magnetostrictive constant, $\lambda_{111}$, of 0.53 $\% \mathrm{Al}-\mathrm{Fe}$ alloy has already been determined in the preceding papers ${ }^{(2)(3)}$, using a linear relationship which holds approximately between the square of the magnetization and the magnetostriction for the [110] or [111] specimen. Next, a different method for the determination of $\lambda_{111}$ in which the difference of magnetostriction between the remanence point and the saturation state is used will be described.

For the [100] specimen, as shown in the preceding paper $^{(1)}$, the difference of magnetostriction between the remanence point and the saturation state is expressed by the equation

$$
\begin{aligned}
\lambda_{k}-\lambda_{s} \simeq & (3 / 2) \lambda_{100}\left\{\left(\beta_{1}^{3} / \sum \beta_{i}\right)-\beta_{1}^{4}-\varepsilon_{1 k}^{m} \beta_{1}^{2}\right\} \\
& -3 \lambda_{111} \beta_{1}^{2}\left(\beta_{2}^{2}+\beta_{3}^{2}\right),
\end{aligned}
$$

which indicates that the value of $\lambda_{k}-\lambda_{s}$ depends on the domain distribution at the remanence point. Furthermore, the difference of magnetostriction between the coercive force point and the saturation state is expressed by the equation

$$
\begin{aligned}
\lambda_{c}-\lambda_{s}= & (3 / 2) \lambda_{100}\left\{\left(\sum_{i} \beta_{i}^{3} \mid \sum_{i} \beta_{i}\right)-\sum_{i} \beta_{\imath}^{4}\right\} \\
& -3 \lambda_{111} \sum_{i<j} \beta_{i}^{2} \beta_{j}^{2},
\end{aligned}
$$

which indicates that the value of $\lambda_{c}-\lambda_{s}$ is independent of the domain distribution and depends on the direction of measurement only. For the [110] specimen $^{(2)}$, the equation corresponding to eq. (2) is expressed by the form

$$
\begin{aligned}
\lambda_{k}-\lambda_{s} \simeq & (3 / 2) \lambda_{100}\left\{\left[\left(\beta_{1}^{3}+\beta_{2}^{3}\right) / \sum_{i} \beta_{i}\right]-\left(\beta_{1}^{4}+\beta_{2}^{4}\right)\right. \\
& \left.-\left(\varepsilon_{1 k}^{m}+\varepsilon_{2 k}^{m}\right) \overline{\beta_{1}^{2}}\right\}-3 \lambda_{111}\left(\overline{\beta_{1}^{2}}\right)^{2},
\end{aligned}
$$

which indicates that the value of $\lambda_{k}-\lambda_{s}$ depends on the domain distribution at the remanence point, like the case of the [100] specimen. On the contrary, for the [111] specimen $^{(3)}$, the equation corresponding to eq. (2) is always expressed by the form

$$
\begin{aligned}
\lambda_{k}-\lambda_{s}= & (3 / 2) \lambda_{100}\left\{\left(\sum_{i} \beta_{2}^{3} / \sum_{i} \beta_{i}\right)-\sum_{i} \beta_{\imath}^{4}\right\} \\
& -3 \lambda_{111} \sum_{2<j} \beta_{i}^{2} \beta_{\jmath}^{2},
\end{aligned}
$$

which is equivalent to eq. (3), and so indicates that the value of $\lambda_{k}-\lambda_{s}$ is independent of the domain distribution. Moreover, eq. (5) holds also for the [100] and [110] specimens, provided that their domain distributions at the remanence points obey Kaya's rule. Then, as mentioned in the preceding papers ${ }^{(1)(2)}$, the terms containing $\varepsilon_{\imath k}^{m} \mathrm{~s}$ in eqs. (2) and (4) which are related to the domain distri- butions at the remanence points are very small; therefore, considering that eq. (5) holds practically for the single crystals which have all sorts of crystallographic orientations, it will be possible to determine the value of $\lambda_{111}$ by using the measured values of $\lambda_{k}-\lambda_{s}$ for the single crystal specimens with various crystallographic orientations. For the single crystal specimen with various orientations, however, the first term on the right side of eq. (5) is small, and also the measured value of $\lambda_{k}-\lambda_{s}$ in eq. (5) for the $[100]$ specimen is very small. Consequently, even if the measured values of $\lambda_{k}-\lambda_{s}$ of the single crystal specimens with various orientations are treated by the method of least squares, it will be found hard to obtain an accurate value of $\lambda_{111}$.

Therefore, in view of the fact that in the [111] specimens both the second term on the right side of eq. (5) and the measured value of $\lambda_{k}-\lambda_{s}$ are largest among the single crystal specimens investigated and the first term on the right side of eq. (5) is negligibly small, the following value for $\lambda_{111}$ was obtained by treating the measured values which were obtained with the three [111] specimens after $\mathrm{TD}$ and $\mathrm{AD}$ by the method of least squares :

$$
\lambda_{111}=-20.5 \times 10^{-6} \text {. }
$$

Moreover, from the slope of the linear portion of the $\lambda^{t}-l^{m}$ curve in the range of wall displacements for the [100] specimen $^{(1)}$ after TD, the magnetostrictive constant $\lambda_{100}$ was obtained as follows :

$$
\lambda_{100}=15.7 \times 10^{-6} \text {. }
$$

In addition, the value of the saturation magnetostriction for a pseudo-isotropic polycrystal, $\bar{\lambda}$, calculated by using eqs. (6) and (7), on the basis of the equation

$$
\bar{\lambda}=(1 / 5)\left(2 \lambda_{100}+3 \lambda_{111}\right) \text {, }
$$

is as follows :

$$
\bar{\lambda}=-6.0 \times 10^{-6} .
$$

This result is comparable with the values of the saturation magnetostriction obtained experimentally for the polycrystal specimen No. 11 after TD and AD, which are

$$
\left(\lambda_{s}\right)_{\mathrm{TD}}=-7.5 \times 10^{-6} \text { and }\left(\lambda_{s}\right)_{\mathrm{AD}}=-8.7 \times 10^{-6} \text {, }
$$

respectively, as shown in Table 4 .

\section{Discussions}

1. Relationship between the behavior of the longitudinal magnetostriction and the method of demagnetization

As an over-all review of the behaviors of the longitudinal magnetostriction in the single crystal and polycrystal specimens of $0.53 \% \mathrm{Al}-\mathrm{Fe}$, the dependence of the magnetostrictive behaviors of these specimens on the method of demagnetization will be described in more detail in reference to the [100] specimen in which such an effect is revealed most conspicuously.

Among the works carried out heretofore on the longitudinal magnetostriction, the results of measurements 
made by Tatsumoto ${ }^{(5)}$ with single crystals of iron containing $1.08 \%$ Si most closely resemble those of the $[100]$ specimens of the author, as described briefly in the preceding paper ${ }^{(1)}$. Tatsumoto's specimens are two kinds of narrow single crystal strips produced from the strips that were cut out parallel or perpendicular to the rolling direction from a rolled silicon steel plate, in which dotted small inclusions are elongated parallel to the rolling direction. In the former, called hereafter the [100] specimen, the longer side of the striated inclusions along which magnetic domains are long and slenderly distributed except for both the ends coincides with the lengthwise direction of the strip, i.e. $[100]$ or $[\overline{100}]$ of the directions of easy magnetization. In the latter, called hereafter the [010] specimen, the longer side of the striated inclusions is nearly at right angles to the lengthwise direction of the strip, coinciding with $[010]$ or $[0 \overline{1} 0]$ of the directions of easy magnetization. Since the dependence of the powder patterns of the two kinds of specimens on the method of demagnetization was not detected, the longitudinal magnetostriction after $\mathrm{AD}$ was measured. On comparison, the $\lambda-I$ curves of Tatsumoto's $[010]$ and $[100]$ specimens turn out to be similar on the whole to those of the $[100]$ specimen of the author obtained after TD and $\mathrm{AD}$, respectively, although there is a difference in the values of saturation magnetostriction. Thus, it may be safely said that the domain distributions of the $[100]$ specimen of the author in the TD and AD states correspond to those of Tatsumoto's [010] and [100] specimens in the unmagnetized states, respectively. The origins of those domain distributions are thought to be as follows. In Tatsumoto's specimen, owing to a particular distribution of solute atoms, a uniaxial magnetic anisotropy is stably induced along a particular direction, that is, the longer side of the striated inclusions, irrespective of the method of demagnetization. In the specimen of the author the domain distribution induced by TD is changed into a different one by $A D$, i. e. the alternating-current demagnetization carried out along the lengthwise direction of the specimen, on account of the alteration of the configuration of solute atoms due to the process of demagnetization, and, as a result of a preference of the domains for the rod axis, the domain distribution in which a uniaxial magnetic anisotropy is induced along the rod axis becomes stable.

Moreover, it has been known in the preceding paper ${ }^{(1)}$ that the $\lambda-I$ curve obtained by Takaki ${ }^{(6)}$ with the $[100]$ single crystal of pure iron under a tensile stress of $680 \mathrm{~g} /$ $\mathrm{mm}^{2}$ resembles that of the [100] specimen of the author obtained after AD. This may be understood from that, in the [100] single crystal of pure iron whose domain distribution in the unmagnetized state is almost uniform, a domain distribution similar to that of the [100] specimen of the author obtained after AD can be induced as well by applying a proper amount of tensile stress along the rod axis of the specimen.

Barbier and Ferlin-Guion ${ }^{(7)}$ carried out a study of the dependence of the domain distribution on the method of

(5) E. Tatsumoto : J. Sci. Hiroshima Univ., A 17 (1953), 229.

(6) H. Takaki : Proc. Phys.-Math. Soc. Japan, 24 (1942), 875.

(7) J. C. Barbier and B. Ferlin-Guion: J. Appl. Phys., 33 (1962), 1226. demagnetization in a ferromagnetic polycrystalline specimen which had not been influenced by the magneticfield-cooling effect. They investigated the difference between the values of magnetization, $I_{x}$ and $I_{y}$, measured along the lengthwise direction of the specimen $(x$ direction), after $A D$ had been carried out along the $x$ direction and at right angles to the $x$ direction ( $y$ direction). They found that the ratio $I_{x} \mid I_{y}$ was larger than unity for a substance of cubic structure which had been annealed to relieve internal strains and had the cubic magnetic anisotropy, and that it was equal to unity within the precision of measurements for a uniaxial substance, such as hexagonal cobalt, and for an unannealed substance of cubic structure in which internal strains remained. Although they made no mention of single crystals, such an anisotropy effect of demagnetization as described above is thought to be more distinctly revealed for single crystals. Therefore, it may be expected that the magnetostriction of the [100] or [110] specimen in the range of wall displacements is affected by this effect. In the specimens of $0.53 \% \mathrm{Al}-$ Fe alloy, however, the author could not detect any such an anisotropy effect in both the single crystals and the polycrystal in the measurements of direction magnetization and magnetostriction along the $x$ direction on the specimens which had been either magnetized to saturation or subjected to $\mathrm{AD}$ along the $x$ direction and, further, subjected to $\mathrm{AD}$ along the $y$ direction. Thus, it is re-confirmed that the specimen of the author has a domain distribution in the unmagnetized state in which the uniaxial magnetic anisotropy is induced besides the cubic one, after being either magnetized to saturation or subjected to AD along the $x$ direction.

The results of measurements on the longitudinal magnetostriction for the single crystal and polycrystal specimens of $0.53 \% \mathrm{Al}-\mathrm{Fe}$ alloy indicate that in the unmagnetized state the relative volumes of the domains whose magnetization vectors preferably are oriented in parallel with the directions of easy magnetization close to the $y$ direction are much larger in the TD state than in the $\mathrm{AD}$ state. Such a domain distribution as revealed in the TD state is thought to be attributable to the effect of directional ordering which is generally induced in the magnetic fieldcooling effect in ferromagnetic cubic solid solution alloys ${ }^{(8)}$. Namely, when subjected to TD at a temperature lower than the Gurie point but high enough as to cause the diffusion of aluminium atoms, such an alloy may have a domain distribution in which the uniaxial magnetic anisotropy energy, $\left(E_{u}\right)_{\mathrm{TD}}$, is added to the cubic magnetic anisotropy energy, $E_{c}$. It is believed that this uniaxial anisotropy differs from position to position in the specimen and the direction of easy magnetization of $\left(E_{u}\right)_{T D}$ at a certain position coincides with the direction of magnetization vector at that position on the way of cooling, which is one of the directions of easy magnetization $\langle 100\rangle$ of $E_{c}$ at that position. Further, as pointed out by Snoek ${ }^{(9)}$, it may be considered that small amounts of carbon atoms lie along

(8) S. Taniguchi and M. Yamamoto : Sci. Rep. Res. Inst. Tohoku Univ., A 6 (1954), 330 ; S.Taniguchi : ibid, A 7 (1955), 269; L. Néel : J. Phys. Radium, 15 (1954), 225; Compt. rend., 241 (1955), 533.

(9) J. L. Snoek : Physica, 8 (1941), 711. 
the cube edges as interstitial impurity atoms and the occupation of interstitial sites along the three directions of the cube edges by carbon atoms is not isotropic, but these atoms tend to occupy the interstitial sites along the cube edge parallel to the direction of the magnetization vector, and so the occupation of those sites by carbon atoms causes a slight increase in length along such a cube edge as described just above.

When the specimen in the above-mentioned state after TD is subjected to either magnetization to saturation along the direction of its rod axis or AD along that direction, we consider that the directions of the magnetization vectors which have been oriented parallel to the $\langle 100\rangle$ directions far from the rod axis are turned into the $\langle 100\rangle$ direction nearest to the rod axis, and this turning leads to the formation of a different distribution of the occupation sites of impurity atoms in such a way as described above, and consequently the distribution of the domains whose magnetization vectors are oriented parallel to the direction of easy magnetization nearest to the rod axis ; that is, a domain distribution analogous to that of Tatsumoto's $[100]$ specimen in the unmagnetized state is established. Thus, it follows that the relative volumes of the domains whose magnetization vectors are oriented parallel to the $\langle 100\rangle$ direction nearest to the rod axis are much larger in the AD state than those in the TD state.

In short, when the specimen of the author is subjected to either magnetization to saturation or $\mathrm{AD}$ along the rod axis of the specimen, the uniaxial anisotropy energy, $\left(E_{u}\right)_{\mathrm{AD}}$, whose direction of easy magnetization coincides with the $\langle 100\rangle$ direction nearest to the rod axis, is thought to be uniformly added to $E_{c}$ and $\left(E_{u}\right)_{\mathrm{TD}}$ over the entire volume of the specimen; also, it can be considered that in average over the entire $\left(E_{u}\right)_{\mathrm{TD}}$ is equivalent to the uniaxial anisotropy energy, $E_{u}$, with a positive constant, while $\left(\mathrm{E}_{u}\right)_{\mathrm{AD}}$ is equivalent to $E_{u}$ with a negative constant. Further, from the above-mentioned results of measurements by the author with the single crystal specimens of $0.53 \% \mathrm{Al}-\mathrm{Fe}$ and those by Tatsumoto ${ }^{(5)}$ with the single crystal strips of $1.08 \% \mathrm{Si}-\mathrm{Fe}$, it may be safely said that the relation

$$
\left|\left(E_{u}\right)_{\mathrm{AD}}\right|>\left|\left(E_{u}\right)_{\mathrm{TD}}\right|
$$

holds between the absolute values of the uniaxial anisotropy energy for the specimens of the author.

It may be concluded experimentally that there is a considerable difference between the magnetic properties of iron containing small amounts of impurity atoms and those of pure iron can be detected more distinctly from the results of measurements on the magnetostriction.

\section{Comparison of the values of the magneto- strictive constants determined by the author and those obtained until now}

The values of the magnetostrictive constants $\lambda_{100}$ and $\lambda_{111}$ of $0.53 \% \mathrm{Al}-\mathrm{Fe}$ alloy have been determined by the methods described above (cf. IV), as shown in eqs. (7) and (6), respectively. To compare these results with the values in the literature, a further description will be made below.

The magnetostrictive constants of $\mathrm{Fe}-\mathrm{Al}$ alloys at ordinary temperatures were systematically investigated by Hall $^{(10)}$ and by Gengnagel(11); however, Gengnagel's results cannot be immediately compared with those of the author, because they were obtained for the alloys containing more than $9.5 \%$ aluminium. The composition dependence of the values of $\lambda_{100}$ and $\lambda_{111}$ for the alloys containing less than $10 \mathrm{wt} \%$ aluminium is shown in Fig. 9, in which Gengnagel's results are also shown for reference. As seen from Fig. 9, the value of $\lambda_{111}$ of $0.53 \% \mathrm{Al}-\mathrm{Fe}$ alloy harmonizes well with the $\lambda_{111}$ vs. composition curve of Hall, but the value of $\lambda_{100}$ of the same alloy of the author deviates lower than the $\lambda_{100}$ vs. composition curve of Hall.

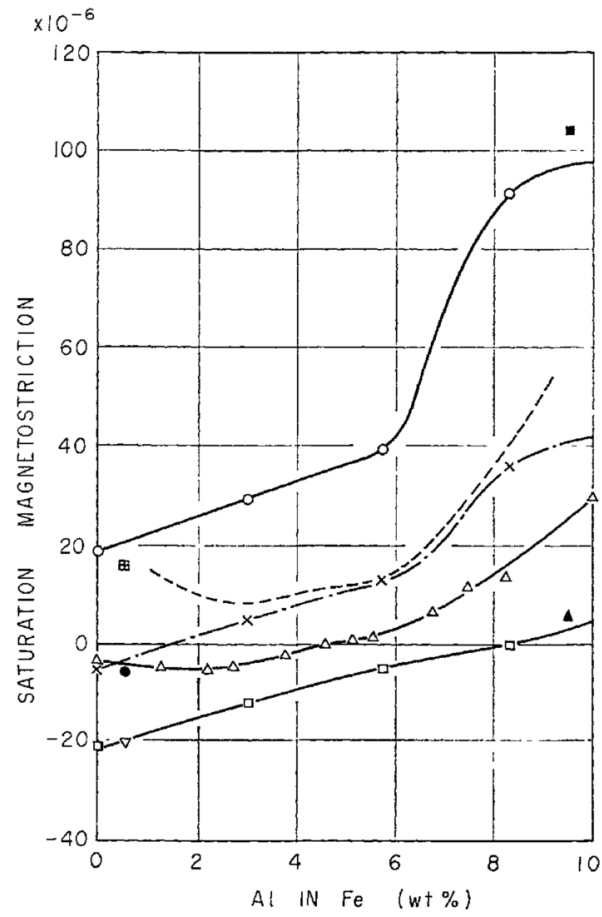

Fig. 9 Composition dependence of the experimental values of $\lambda_{100}, \lambda_{111}$ and $\lambda_{s}$ obtained by several authors, and of the values of $\bar{\lambda}$ and $\lambda_{100}$ calculated by eq. $(7)$, in the $\mathrm{Fe}-\mathrm{Al}$ system

Symbols are used as follows :

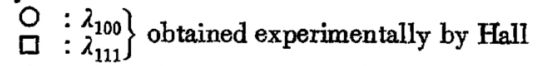

$$
\begin{aligned}
& \Delta: \lambda_{s} \text { obtained experimentally by Masumoto and } \\
& \text { Saito } \\
& x: \bar{\lambda} \text { calculated by eq. (7) from Hall's data } \\
& \text { - - : }: \lambda_{100} \text { calculated by eq. (7) from the data of Hall } \\
& \text { and Masumoto and Saito }
\end{aligned}
$$

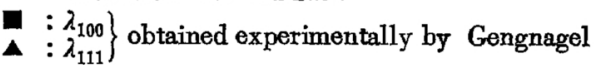

$$
\begin{aligned}
& \text { 田: } \lambda_{100} \text { obtained experimentally by the present } \\
& \left.\begin{array}{c}
: \\
: \\
\lambda_{s}
\end{array}\right\} \text { author }
\end{aligned}
$$

Since the data available on the magnetostrictive constants of $\mathrm{Fe}-\mathrm{Al}$ alloys are restricted to those described above, the measured values of the saturation magnetostriction of polycrystalline $\mathrm{Fe}-\mathrm{Al}$ alloys are compared here. Investigations on the magnetostriction of the polycrystalline $\mathrm{Fe}-\mathrm{Al}$ alloys in the low composition range of aluminium have in detail been made by Honda and his

(10) R. C. Hall : J. Appl. Phys., 30 (1959), 816.

(11) H. Gengnagel : Naturwiss., 45 (1958), 81. 
co-workers ${ }^{(12)}$ and by Masumoto and Saito ${ }^{(13)}$. Since the value of the saturation magnetostriction for the polycrystal specimen of $0.53 \% \mathrm{Al}-\mathrm{Fe}$ alloy of the author harmonizes better with the saturation magnetostriction vs. composition curve of the latter than that of the former, the composition dependence of the saturation magnetostriction $\lambda_{s}$ for the latter is also shown in Fig. 9. To compare the data of Masumoto and Saito with the values obtained from the results of measurements for single crystals, the value of the saturation magnetostriction for a pseudo-isotropic polycrystal, $\bar{\lambda}$, which was calculated from eq. (8) by use of the values of the magnetostrictive constants obtained by Hall, is also plotted against the composition in Fig. 9. In the low composition range of aluminium, this $\bar{\lambda}$ vs. composition curve shows a monotonic increase with increase in aluminium content. On the contrary, the results of measurements of Masumoto and Saito indicate that in the composition range from zero to about $2 \% \mathrm{Al}$ the value of $\lambda_{s}$ decreases with increase in aluminium content, and subsequently, after arriving at a minimum value at a composition near $2 \% \mathrm{Al}$, it increases up to about $12 \% \mathrm{Al}$ (not shown in Fig. 9). Considering that the value of $\lambda_{111}$ increases monotonically with increase in aluminium content in this composition range, as seen in the measured values of Hall, the $\lambda_{s}$ vs. composition curve of Masumoto and Saito will not harmonize with the $\bar{\lambda}$ vs. composition curve obtained from the calculation, unless the value of $\lambda_{100}$ decreases temporarily, with increase in aluminium content, less than that corresponding to pure iron. Since the determination of $\lambda_{111}$ is scarcely affected by the domain distribution of the specimen in the unmagnetized state and, further, the value of $\lambda_{111}$ of $0.53 \%$ Al-Fe alloy of the author harmonizes well with the $\lambda_{111}$ vs. composition curve of Hall, an attempt has been made to calculate $\lambda_{100}$ for the alloys in this composition range; namely, in order to calculate $\lambda_{100}$ from eq. (8), the value of the saturation magnetostriction obtained experimentally by Masumoto and Saito has been used in place of $\bar{\lambda}$ of eq.

(12) K. Honda, H. Masumoto, Y. Shirakawa and T. Kobayashi : J.Japan Inst. Metals, 12 (1948), No. 7 12 ; Sci. Rep. Res. Inst. Tohoku Univ., A 1 (1949), 341.

(13) H. Masumoto and H. Saito : Sci. Rep. Res. Inst. Tohoku Univ., A 4 (1952), 338
(8), together with the value of $\lambda_{111}$ known from the $\lambda_{111}$ vs. composition curve of Hall. By plotting the values of $\lambda_{100}$ calculated in this way against the composition, such a $\lambda_{100}$ vs. composition curve as shown by a dashed line in Fig. 9 has been obtained. It is evident that this curve harmonizes with the value of $0.53 \% \mathrm{Al}-\mathrm{Fe}$ alloy of the author.

Thus, it can be expected that the $\lambda_{100}$ vs. composition curve of $\mathrm{Fe}-\mathrm{Al}$ alloys has a minimum value near the composition of about $3 \% \mathrm{Al}$, although it is necessary to determine experimentally the values of $\lambda_{100}$ with single crystal specimens of the alloys with higher aluminium concentrations.

\section{Summary}

From the measurements of the longitudinal magnetostriction of the [210] single crystal and a polycrystal of iron containing $0.53 \% \mathrm{Al}$, it has been made clear that the magnetostrictive behavior of the former depends strongly on the method of demagnetization and as a whole shows an intermediate behavior between the [100] and [110] specimens, and that the behavior of the latter is by and large similar to that of the [110] specimen after TD, although there is a considerable difference between their behaviors after AD. Both the specimens show that the difference of magnetostriction between the remanence point and the saturation state is independent of the method of demagnetization. In consideration of the difference of magnetostriction between the above two states, for the determination of the magnetostrictive constant $\lambda_{111}$ has been made. The value of $\lambda_{111}$ thus obtained is $-20.5 \times 10^{-6}$. Finally, consideration has been given to the difference between the effects produced by TD and AD for the specimens of the author and also to a comparison between the values of the magnetostrictive constants determined in the present study and those of $\mathrm{Fe}-\mathrm{Al}$ alloys reported heretofore.

\section{Acknowledgment}

The author wishes to express his sincere thanks to Prof. M. Yamamoto for his guidance. 